\title{
Mixed Integer Linear Programs for Blocking and No Wait Job Shop Scheduling Problems in Robotic cells
}

\author{
Saad Louaqad \\ Laboratoire des Technologies Innovantes \\ ENSAT - University ABDELMALEK ESSADI \\ 90000 Tanger, Morocco
}

\author{
Oulaid Kamach \\ Laboratoire des Technologies Innovantes \\ ENSAT - University ABDELMALEK ESSADI \\ 90000 Tanger, Morocco
}

\begin{abstract}
This paper studies the problem of scheduling Job shops in robotic cells with no intermediate buffers, called No Wait Blocking Transport Job Shop Scheduling Problem (NWBT JSSP). This problem is an extension of the classical job shop problem. No Wait Blocking Transport job shop problems arise in many realistic production environments. To tackle this problem, we developed a Mixed Integer Linear Program and proposed a constructive heuristic based on priority rules. The MILP model has been used to solve optimally problems with as many as ten jobs, ten machines and three robots. Computational results on hypothetically generated test problems are discussed and suggestions of future research projects are proposed.
\end{abstract}

\section{General Terms}

Optimization Problems, Manufacturing Scheduling

\section{Keywords}

Job Shop Scheduling problem, Transport Constraint, Blocking Constraint, No Wait Constraint, Mixed Integer Linear Program

\section{INTRODUCTION}

The Job Shop Scheduling Problems are well-known combinatorial optimization problems, which consist of a finite number of jobs and machines. Each job consists of a set of operations that has to be processed in a given order, on a set of known machines, and where each operation has a known processing time. No machine can process more than one operation at the same time. The objectives usually considered in JSSPs are the minimization of Makespan.

Considerable research has been devoted to this problem in the literature. An overview of history and main techniques used along with their reported results on the available benchmarking problems for JSSP can be found in [1].

Several problems in practice cannot be modeled as (classical) Job Shop, due to additional features like storage space and transportation constraints. The classical model supposes that storage space has an infinite capacity and transport operations from one machine to another take almost no time. In many practical cases, the number of buffers is limited and transports need to be considered for various reasons. Buffers may be expensive or inadequate for technological or process reasons. Transports may take a considerable amount of time, or only a limited number of mobile devices are available to execute transports operations.

The jobs shop problems with transportation, blocking and no wait constraints are met for example in factories with robotic cells. A robotic cell is a flow-shop or job-shop scheduling problem in which the jobs must be transported from machine to machine. Transports are performed by one or more robots. We have to assign the transport operations to the robots and to schedule both the machine and robot operations. Usually there is no buffer or buffers with only limited capacity to store the jobs outside the machines or the robots. This means that our scheduling must deal with blocking and no wait constraints.

Job shop models with blocking constraints (BJSSP) have been discussed by several authors. [2] give a survey on machine scheduling problems with blocking and no-wait constraints. [3] describes several applications of machine scheduling with blocking and nowait in process and reviews the computational complexity of a variety of related problems. Several researchers studied the Blocking Job Shop Scheduling problem (BJSSP) and the No Wait Job Shop Scheduling Problem (NWJSSP). [4] and [5] formulate these problems by means of alternative graphs.|7| develop a genetic algorithm for solving no-wait and Blocking Job Shop problems and [8] and [9] introduce a local search approach for the generalized Blocking Job Shop problem with application in automated warehouses. [6] study a multi-resource job shop problem with blocking constraints. [10] propose a tabu search algorithm to solve the BJSSP for cyclical scheduling. [11] proposes a combination of a branch and bound algorithm with alternative graphs and develops two methods based on genetic algorithms to solve the BJSSP.

Scheduling problems with blocking constraints appear more difficult to solve than the classical job shop. This is due, first, to the fact that a feasible partial schedule for BJSSP cannot always be extended to a feasible complete schedule. In fact, [4] and [5] established that deciding whether this is possible is NP complex. Secondly, it is not straightforward to construct feasible neighbor solutions in a local search approach as moves based on simple swaps of adjacent operations typically yield infeasible schedules. Applications related to the BJS problem have been reported in the processing and logistics industries, such as scheduling for the manufacturing of concrete blocks by [12] , steelmaking by [13] , chemical batch production by [14], container handling at a port by [15] and railway networks by [16].

Several researchers have devoted to study job shop scheduling problems with transportation constraints in various systems. How- 
ever, the progress is limited as this kind of problem is difficult to solve even for simplified and small size cases.

[17] integrated transport constraints in the scheduling problem with one robot. [18] proposed a dynamic programming approach to construct optimal machine and vehicle schedules. [19] developed a mixed integer programming (MIP) formulation raising this constraint on the vehicles. [20] and [21] elaborated a genetic algorithm. [22] and [23] proposed, respectively, neural networks and tabu search approaches. [24] described a hybrid method composed of a genetic algorithm for the scheduling of machines and a heuristic for the scheduling of vehicles. [25] and [26] considered a job shop problem with several robots, with fixed operation times and fixed assignment of machine for each job's operation. [27] studied a two machines flow shop scheduling problem with intermediate transportation with a single transporter. [28] considered a cyclic hoist scheduling problem with a single hoist, but without assignment problem. [29] used a mixed integer linear program (MILP) to find optimal solutions for the Flexible Manufacturing Systems Scheduling Problem with one vehicle. [30] proposed a polynomial algorithm for finding the optimal cycle in a robotic cell with production of identical parts. [31] studied coupled task problem and one-machine robotic cell problems. It reported new algorithmic procedure for this problem with or without tolerances on the distance. [32] applied a decomposition method where the master problem (scheduling) is modeled with constraint programming and the subproblem (conflict free routing) with mixed integer programming.

To the best of our Knowledge there is no research that addressed the problem of job shop scheduling that take into account transfer time between machine performed by a limited number of robot and the non existence of buffers between machines that lead to blocking and no wait constraints.

Two common approaches to tackle the scheduling problems are the utilization of mathematical programming and heuristic approaches [33]. By Mathematical programming methods we formulate the scheduling problems as a mixed integer linear programming (MILP) problem and then tries to solve the formulated problem by a general purpose mixed integer linear programming solver. This methods are usually suitable for small instances. However and due to the great advance recently obtained in capacity of computers and creation of fast optimization software, presentation of MILP models is becoming more and more interesting among the researchers.

In this paper, we propose two methods to address the Job Shop Scheduling Problem with No wait, Blocking and Transportation Constraints (NWBT JSSP). We develop first a Mixed Integer Linear programming model based on the model of [34] and secondly we construct an heuristic based on priority rules.

This paper is structured as follows. In the next section, we will present definitions and notations associated to our model. After that, in Section 3, we describe our integer programming formulation for the NWBT JSSP. In Section 4, we present the algorithm of our proposed heuristic based on priority rules. Section 5 reports the computational results we obtained by solving the problem through the Mathematical model described in this paper with the help of MILP solver IBM ILOG Cplex V12. Finally, Section 6 gives some interesting conclusions and future studies.

\section{PROBLEM DEFINITION}

We consider a job shop problem with several transport robots and no buffers. In this problem, a set of $\mathrm{n}$ jobs $\left\{J_{1}, J_{2}, \ldots, J_{n}\right\}$ are processed on a set of m machines $\left\{M_{1}, M_{2}, \ldots, M_{m}\right\}$ and trans- ported by a set of $\mathrm{k}$ robots $\left\{r_{1}, r_{2}, \ldots, r_{k}\right\}$. Transportation times are robot-independent. Every job $J_{i}$ require an operation order, $J_{i}=\left\{O_{i 1}, O_{i 2}, . ., O_{i n_{i}}\right\}$, that must be executed according to its manufacture process. Operation $O_{i j}$ of the job $J_{i}$ requires the exclusive use of $M_{l}(l \in\{1,2, \ldots, m\})$ for an uninterrupted duration $p_{i j}$, its processing time; the preemption is not allowed; each machine can process only one job at a time; the machine which execute the operation $O_{i j}$ is denoted as $M_{i j}$.

In addition, we consider transportation operations between two machines. Consider two successive operations of the same job $O_{i j}$ and $O_{i j+1}$ to execute in two machines $M_{i j}$ and $M_{i j+1} . T_{i j}$ is used to denote transport operation of job $J_{i}$ from machine $M_{i j}$ to machine $M_{i j+1}$. Each robot can handle at most one job at one time. Loaded transfer times does not depend on the job transported, but only on the travel routes and the robot which perform the transportation operation. This times are given by $C_{p, l}^{r}$ where $r$ represents the robot and $(p, l)$ represents the route between machine $M_{p}$ and $M_{l}$. It is assumed that the triangle inequality is satisfied:

$\forall p, l \in\{1,2, \ldots, m\}$ machine indexes.

$\forall r \in\left\{r_{1}, r_{2}, \ldots, r_{k}\right\}$

$$
C_{p, h}^{r}+C_{h, l}^{r} \geq C_{p, l}^{r}
$$

(1) means that the direct way between two machines is at least as short as the detour through a third machine. Otherwise, the robot always takes the shorter way through the third machine.

Note that a sequence of loaded transport operations indirectly induces necessary empty moves. Empty transfer time from machine $M_{p}$ to $M_{l}$ is denoted $V_{p, l}^{r}$. It is assumed that:

$\forall r, r^{\prime} \in\left\{r_{1}, r_{2}, \ldots, r_{k}\right\}$

$\forall p, l \in\{1,2, \ldots, m\}$ machine indexes

$$
\left\{\begin{array}{l}
V_{p, p}^{r}=0 \\
V_{p, h}^{r}+V_{h, l}^{r} \geq V_{p, l}^{r} \\
V_{p, l}^{r} \leq C_{p, l}^{r^{\prime}}
\end{array}\right.
$$

The first assumption means that no empty transfer time is considered if a robot waits at the same machine the next transportation operation. The second one is the triangular inequality for empty moves. The third one means that empty transfers between two machines by a robot $r$ take less time than loaded transfers between this two machines by another robot $r^{\prime}$. (It is also valid if $r=r^{\prime}$ ). In the other hand, we consider the blocking constraint because there is no machine buffer. This means that after finishing its processing on the machine, a job has to stay there until it is unloaded by the robot. During this stay, the machine is blocked and not available for processing any other job. We also consider the no wait constraint that means if the robot transporting the job $J_{i}$ reaches machine $M_{i j}$, the operation $O_{i j}$ must start immediately without any interruption. The scheduling problem objectives are:

- To determine the starting time for each machine operation $O_{i j}$

- To assign a handling robot to each transport operation $T_{i j}$ and to determine its starting time

- To minimize the Makespan denoted $C_{\max }=\max \left(C_{i}\right)$ where $C_{i}$ denotes the completion time of the last operation of job $J_{i}$.

All data are assumed to be non-negative integers.

\section{MIXED INTEGER PROGRAMMING MODEL}

This section presents the MILP model to formulate NWBT JSSP. Our following formulation is based on the model of [34]. We used 
their ideas to model the Job Shop Scheduling Problem with No Wait, Blocking and Transportation constraints.

To model the Job Shop Scheduling Problem with No Wait, Blocking and Transportation constraints, the following notations are used.

List of parameters :

- $p_{i j}$ : the processing time of operation $O_{i j}$

- $C_{p l}^{r}$ : on load transfer time of robot $r$ between machine $p$ and machine $l$

- $V_{p l}^{r}$ : empty transfer time of robot $r$ between machine $p$ and machine $l$

- $H$ a large number

List of variables:

$-d_{i j}$ : the start time of machine operation $O_{i j}$

- $f_{i j}$ : the completion time of machine operation $O_{i j}$. (Time when operation $O_{i j}$ leave machine $M_{i j}$

$-d^{\prime}{ }_{i j}$ : the start time of transport operation $T_{i j}$

- $f_{i j}^{\prime}$ :the completion time of transport operation $T_{i j}$

List of decision variables:

- $\alpha_{i j ; l q}$ : Binary variable that takes value 1 if $O_{i j}$ is processed before $O_{l q}$ and 0 otherwise.

$-\beta_{i j ; r_{s}}$ : Binary variable that takes value 1 if $T_{i j}$ require processing on robot $r_{s}$, and 0 otherwise.

- $\delta_{i j ; l q}$ : Binary variable that takes value 1 if $T_{i j}$ is processed before $T_{l q}$, and 0 otherwise.

- $\lambda_{i j ; l q}$ : Binary variable that takes value 1 if $T_{i j}$ and $T_{l q}$ are processed by the same robot, and 0 otherwise.

- $\sigma_{i j ; l q}^{r_{s}}$ : Binary variable that takes value 1 if $T_{i j}$ and $T_{l q}$ are processed by the same robot $r_{s}$, and 0 otherwise.

The problem is formulated as follows :

\section{Minimize $C \max$ \\ Subject to:}

- Finish time of machine operations:

For $i \in[1, n] ; j \in[1, m]$

$$
f_{i j} \geq d_{i j}+p_{i j}
$$

- Precedence Constraints between transport operations and machine operations:

For $i \in[1, n] ; j \in[1, m-1]$

$$
\left\{\begin{array}{l}
f^{\prime}{ }_{i j}=d_{i j+1} \\
d_{i j+1}=d^{\prime}{ }_{i j}+\sum_{s=1}^{k} \beta_{i j, r_{s}} C_{M_{i j} M_{i j+1}}^{r_{s}}
\end{array}\right.
$$

- Precedence constraints between machine operations and transport operations:

For $i \in[1, n] ; j \in[1, m-1]$

$$
d^{\prime}{ }_{i j}=f_{i j}
$$

- Disjunctive constraints between machine operations:

For $i, l \in[1, n] ; j, q \in[1, m] / M_{i j}=M_{l q}$

$$
\left\{\begin{array}{l}
d_{i j} \geq f_{l q}-H * \alpha_{i j ; l q} \\
d_{l q} \geq f_{i j}-H *\left(1-\alpha_{i j ; l q}\right) \\
\alpha_{i j ; l q}+\alpha_{l q ; i j}=1
\end{array}\right.
$$

Table 1. Number of constraints generated by our formalization

\begin{tabular}{|r|r|}
\hline Constraint & Constraint Numbers \\
\hline$(3)$ & $n m$ \\
\hline$(4.1)$ & $n(m-1)$ \\
$(4.2)$ & $n(m-1)$ \\
\hline$(5)$ & $n(m-1)$ \\
\hline$(6.1)$ & $1 / 2\left(m n^{2}-m n\right)$ \\
$(6.2)$ & $1 / 2\left(m n^{2}-m n\right)$ \\
$(6.3)$ & $1 / 2\left(m n^{2}-m n\right)$ \\
\hline$(7)$ & $n \times(m-1)$ \\
\hline$(8.1)$ & $k\left((n m-n)^{2}-(n m-n)\right)$ \\
$(8.2)$ & $k\left((n m-n)^{2}-(n m-n)\right)$ \\
$(8.3)$ & $k\left((n m-n)^{2}-(n m-n)\right)$ \\
$(8.4)$ & $\left((n m-n)^{2}-(n m-n)\right)$ \\
$(8.5)$ & $\left((n m-n)^{2}-(n m-n)\right)$ \\
$(8.6)$ & $\left((n m-n)^{2}-(n m-n)\right)$ \\
$(8.6)$ & $\left((n m-n)^{2}-(n m-n)\right)$ \\
\hline
\end{tabular}

Table 2. Number of variables generated by our formalization

\begin{tabular}{|r|r|}
\hline Variable & Variable Numbers \\
\hline$d_{i j}$ & $n m$ \\
\hline$f_{i j}$ & $n m$ \\
\hline$d_{i j}^{\prime}$ & $n(m-1)$ \\
\hline$f_{i j}^{\prime}$ & $1 / 2\left(n^{2}-n\right)$ \\
\hline$\alpha_{i j ; l q}$ & $k n(m-1)$ \\
\hline$\beta_{i j ; r_{s}}$ & $(n m-n)(n m-n-1)$ \\
\hline$\delta_{i j ; l q}$ & $(n m-n)(n m-n-1)$ \\
\hline$\lambda_{i j ; l q}$ & $k(n m-n)(n m-n-1)$ \\
\hline$\sigma_{i j ; l q}^{r_{s}}$ & $k$ \\
\hline
\end{tabular}

- Robot assignment constraints:

For $i \in[1, n] ; j \in[1, m-1], s \in[1, k]$

$$
\sum_{s=1}^{k} \beta_{i j, r_{s}}=1
$$

- Disjunctive constraints between robot:

For $i, l \in[1, n] ; j, q \in[1, m-1] ; s \in[1, k]$

(3) Ensures that each operation is processed at least for its process duration

(4) ensures that each machine operation starts immediately after the finish of the transport operation that precede it.

(5) As there is no buffer in machines, transport operation starts immediately when the job leaves a machine .

(6) ensures that each machine process one operation at a time.

(7) ensures that each transport operation is performed by only one Robot.

8) ensures that each robot process one operation at a time. no two transport operations are performed by the same robot at any time

The complexity of a mathematical model depends on the number of its variables and constraints. Table [1] and Table [2] presents the total number of variables and constraints generated by the proposed MILP model.

Examining the above tables shows that the complexity of our mathematical model is significantly increased by the introduction of additional indexes to address the problem of machines and 


$$
\left\{\begin{array}{l}
\sigma_{i j ; l q}^{r_{s}} \geq 1-\left(1-\beta_{i j, r_{s}}\right) H-\left(1-\beta_{l q, r_{s}}\right) H \\
\sigma_{i j, l q}^{r_{s}} \leq \beta_{i j, r_{s}} \\
\sigma_{i j ; l q}^{r_{s} ; l q} \leq \beta_{l q, r_{s}} \\
\lambda_{i j ; l q}=\sum_{s=1}^{k} \sigma_{i j ; l q}^{r_{s}} \\
d^{\prime}{ }_{i j} \geq d^{\prime}{ }_{l q}+\sum_{s=1}^{k} \beta_{l q, r_{s}}\left(C_{M_{l q} M_{l q+1}}^{r_{s}}+V_{M_{l q+1} ; M_{i j}}^{r_{s}}\right)+\left(\lambda_{i j ; l q}-1\right) H-\delta_{i j ; l q} H \\
d_{l q}^{\prime} \geq d^{\prime}{ }_{i j}+\sum_{s=1}^{k} \beta_{i j, r_{s}}\left(C_{M_{i j} M_{i j+1}}^{r_{s}}+V_{M_{i j+1} ; M_{l q}}^{r_{s}}\right)+\left(\lambda_{i j ; l q}-1\right) H+\left(\delta_{i j ; l q}-1\right) H \\
\delta_{i j ; l q}+\delta_{l q ; i j}=1
\end{array}\right.
$$

Table 3. Calculation of Number of constraints and variables generated by our formalization $i$

\begin{tabular}{|l|l|l|l|}
\hline Instance & $\begin{array}{l}\text { Problem Size } \\
n \times m \times k\end{array}$ & $\begin{array}{l}\text { Variable } \\
\text { Number }\end{array}$ & $\begin{array}{l}\text { Constraint } \\
\text { Number }\end{array}$ \\
\hline Inst 3.1 & $3 \times 3 \times 1$ & 129 & 270 \\
\hline Inst 3.2 & $3 \times 3 \times 2$ & 165 & 360 \\
\hline Inst 3.3 & $3 \times 3 \times 3$ & 201 & 450 \\
\hline Inst 4.1 & $4 \times 4 \times 1$ & 470 & 1060 \\
\hline Inst 4.2 & $4 \times 4 \times 2$ & 614 & 1456 \\
\hline Inst 4.3 & $4 \times 4 \times 3$ & 758 & 1852 \\
\hline Inst 5.1 & $5 \times 5 \times 1$ & 1260 & 2915 \\
\hline Inst 5.2 & $5 \times 5 \times 2$ & 1660 & 4055 \\
\hline Inst 5.3 & $5 \times 5 \times 3$ & 2060 & 5195 \\
\hline Inst 6.1 & $6 \times 6 \times 1$ & 2787 & 6516 \\
\hline Inst 6.2 & $6 \times 6 \times 2$ & 3687 & 9126 \\
\hline Inst 6.3 & $6 \times 6 \times 3$ & 4587 & 11736 \\
\hline Inst 7.1 & $7 \times 7 \times 1$ & 5411 & 12712 \\
\hline Inst 7.2 & $7 \times 7 \times 2$ & 7175 & 17878 \\
\hline Inst 7.3 & $7 \times 7 \times 3$ & 8939 & 23044 \\
\hline Inst 8.1 & $8 \times 8 \times 1$ & 9564 & 22520 \\
\hline Inst 8.2 & $8 \times 8 \times 2$ & 12700 & 31760 \\
\hline Inst 8.3 & $8 \times 8 \times 3$ & 15836 & 41000 \\
\hline Inst 9.1 & $9 \times 9 \times 1$ & 15750 & 37125 \\
\hline Inst 9.2 & $9 \times 9 \times 2$ & 20934 & 52461 \\
\hline Inst 9.3 & $9 \times 9 \times 3$ & 26118 & 67797 \\
\hline Inst 10.1 & $10 \times 10 \times 1$ & 24545 & 57880 \\
\hline Inst 10.2 & $10 \times 10 \times 2$ & 32645 & 81910 \\
\hline Inst 10.3 & $10 \times 10 \times 3$ & 40745 & 105940 \\
\hline
\end{tabular}

robots allocation.

The table [3] provides some numerical examples for various sizes of instances. the obtained values are very significant.

\section{HEURISTIC}

\subsection{Blocking Situation}

In this section, we will look for situations that could lead to blocking states in order to avoid them during the construction of our algorithm. For this purpose, we will identify blocking situations by using a graph $G_{s}=(M, J)$ that we will call the graph of last scheduled operations. This graph is defined below: Consider the graph of last scheduled operations $G_{s}=(M, J)$. A set of vertices $M$ represents machines; A set of arcs $J$ represents the last scheduled operations $O_{i j}$ of job $J_{i}$. The starting point of the last scheduled operation $O_{i j}$ of job $J_{i}$ is the machine $M_{i j}$ and its end point is the machine $M_{i j+1}$.

Consider for example a problem with 5 machines $\left\{M_{1}, M_{2}, \ldots, M_{5}\right\}$ and 4 jobs $\left\{J_{1}, J_{2}, J_{3}, J_{4}\right\}$. The last scheduled

operations are: $J_{1}: M_{1}-->M_{2} ; J_{2}: M_{2}-->M_{3}$; $J_{3}: M_{3}-->M_{1}, J_{4}: M_{4}-->M_{5}$. The associated graph $G_{s}$ is modeled as follows:
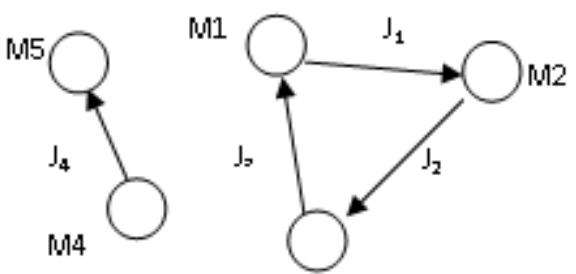

MI3

Fig. 1. Associated graph of last scheduled operations

Following the topology of $G_{s}=(M, J)$, the graph contains a cycle of length $p=3$ ( $p$ equals the number of jobs that forms the cycle. Under this cycle, the necessary condition of blocking (C1) is satisfied because job $J_{1}$ that is processed on the machine $M_{1}$ cannot pass to machine $M_{2}$ occupied by the job $J_{2}$, as well as for job $J_{2}$ that is processed on machine $M_{2}$ cannot pass to machine $M_{3}$ occupied by job $J_{3}$ as well as for job $J_{3}$ that is processed on machine $M_{3}$ cannot pass to machine $M_{1}$ occupied by the job $J_{1}$. Blocking condition (C1) can be formulated as follows: "Systems may confront a blocking situation if the graph of the last scheduled operations $G_{s}=(M, J)$ contains a cycle of length $p \geq 2$." Thereafter, we will check if the condition $(\mathrm{C} 1)$ is a sufficient condition to blocking situation in BNWT JSSP.

1st Case : $p=2$

Consider the graph $G_{s}$ with a cycle of length $p=2$.
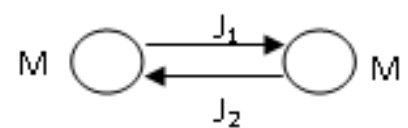

Fig. 2. Graph with a cycle of length $p=2$

When the processing of job $J_{1}$ on machine $M_{1}$ has finished, job $J_{1}$ remains blocked on machine $M_{1}$ until its transportation operation $T_{1}$ from $M_{1}$ to $M_{2}$ start. We assume that a robot $r_{1}$ is available and will be assigned to transportation operation $T_{1}$. This operation can start and liberate the machine $M_{1}$. No wait condition imply that the processing of job $J_{1}$ on machine $M_{2}$ must start immediately 
after the termination of transport operation $T_{1}$ otherwise $J_{1}$ cannot be transported by $r_{1}$ and then machine $M_{1}$ remains blocked. $M_{2}$ is blocked by $J_{2}$ and must be liberated before the termination of transport operation $T_{1}$. Therefore, we must have a second robot to execute transport operation $T_{2}$ of $J_{2}$ before that $J_{1}$ arrives at $M_{2}$.

Proposition 1: If the graph of last scheduled operations $G_{s}=(M, J)$ contains a cycle of length $p=2$, the system is eternally blocked if it has one handling robot $(k=1)$ and partially blocked if $k \geq 2$.

Second Case: $p \geq 3$

Consider the graph $G_{s}$ which a cycle of length $p \geq 3$

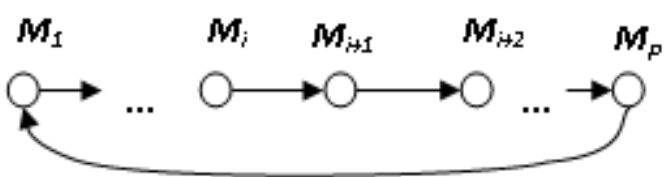

Fig. 3. graph with a cycle of length $p \geq 3$

We assume that a robot $r_{1}$ is available. Robot $r_{1}$ will be assigned to operation $T_{i}$. Before that operation $T_{i}$ has finished, operation $T_{i+1}$ must start. For this reason, we have a second robot $r_{2}$ which will be assigned to operation $T_{i+1}$.

Assumption 1: Assume that operation $T_{i+1}$ starts at the time that $T_{i}$ finishes: $d^{\prime}{ }_{i+1}=f^{\prime}{ }_{i}$. At the time $f^{\prime}{ }_{i}, r_{1}$ arrives in front of machine $M_{i+1}$ and unload job $J_{1}$. Then it can drive empty to machine $M_{i+2} . r_{1}$ reaches $M_{i+2}$ at a time equal to $f_{i}^{\prime}+V_{M_{i+1} M_{i+2}}^{r_{1}}$. The robot $r_{2}$ which is performing transport operation $T_{i+1}$ reaches machine $M_{i+2}$ at a time equal to $d^{\prime}{ }_{i+1}+C_{M_{i+1} M_{i+2}}^{r_{2}}$. According to (2) and (Assumption 1) we have: $f^{\prime}{ }_{i}+V_{M_{i+1} M_{i+2}}^{r_{1}} \geq d^{\prime}{ }_{i+1}+C_{M_{i+1} M_{i+2}}^{r_{2}}$. This means that the robot $r_{1}$ reaches machine $M_{i+2}$ and liberate this machine before that the robot $r_{2}$ which is executing $T_{i+1}$ reaches this machine and so on until the blocking cycle is totally liberated.

Proposition 2: If the graph of last scheduled operations $G_{s}$ contains a cycle of length $p \geq 3$, the system is eternally blocked if it has a single handling robot $(k=1)$ and partially blocked if $k \geq 2$.

\subsection{The proposed Heuristic}

In this subsection we propose a heuristic dedicated to the BNWT JSSP. During construction of the algorithm, we complete iteratively a partial schedule $S$. $U$ denotes the set of non-scheduled operations. At each iteration of the heuristic, two operations are selected, namely a machine operation and a transportation operation. When a machine operation is chosen on the basis of selection rules, the transportation operation which precedes this machine operation is automatically selected and a robot is assigned according to another priority rules.

The Iteration starts with the construction of the set $\mathrm{E}$ of eligible machine operations. An operation is eligible for partial schedule $S$ if it is a non scheduled operation that can start without violating any constraints.
- Rule 1: Eligible operations $S\left(M_{p}\right)$ that need to be processed on machine $M_{p}$ cannot be scheduled as long as machine $M_{p}$ is occupied by another job. These operations will be eliminated from the set $E$.

- Rule 2 : Eligible operations that could lead to eternal blocking situations or even a cycle in the graph $G_{s}(M, J)$ with $p \geq 3$ will also be eliminated from the set $E$.

After the determination of eligible operations, it remains to appoint the machine operation to be scheduled. For this purpose, we associate each operation $O_{i j} \subset E$ to a pair $\left(M_{p}, g\right)$ where $M_{p}=M_{i j}$ and $g$ is the total time of operations $S\left(M_{p}\right) \subset U$, $g$ can be seen as the weight of $M_{p}$ on the set of non scheduled operations $U$.

Selection rules of machine operations: Machine operation to be scheduled is the one associated to a pair $\left(M_{p}, g\right)$ with the largest $g(g=\operatorname{gmax})$

- Rule 3: If we have on the set $\mathrm{E}$ two or more operations with the same pair $\left(M_{p}, g \max \right)$, we choose the operation that has the longest queue.

- Rule 4: If we have on the set E two operations with the same gmax but with different machines, we schedule that terminate first.

Selection rules of robots:

- Rule 5: To select the robot that will perform the transportation operation, we opted to choose the robot which provides the minimal completion time of the transportation operation, which involves exploring all robots for each assignment.

- Rule 6: In the case of a cycle of length $p=2$, we choose the robot that has the earliest availability time. This time correspond to the empty robot arrival time at the departure machine for the loaded move.

\section{COMPUTATIONAL RESULTS}

There is no comparable mathematical model in the literature to compare with the proposed MILP model. So in this section, 24 hypothetical test problems have been solved only to investigate behavior of proposed MILP model in dealing with problems with different sizes. The different instances have been generated as follows: There is 7 problem sizes ranging from $(3 * 3)$ up to $(10 * 10)$. The processing times are randomly distributed over $(10,100)$. For each problem size, 3 instances with one robot, two similar robot and three similar robot have been generated. A machine layout has been defined. Transport times are correlated to the distances between different machines.

The problems have been solved with CPLEX 12.6 on an Intel(R) Core (TM) $i 7-62720 Q M$ CPU @ 2.2GHz computer with 8 GB memory, running Windows 64 bit.

The results obtained by the proposed MILP model are shown in the Table [4].

The results show that the proposed mathematical model can find optimal solution to the problems with size $(7 * 7 * 2)$. Also when the problem size extends, the gap between best possible bound and MILP solution increases as a consequence.

It is Observed that in some instances even with the introduction of an additional robot, the makespan is not improved. By solving our problem, we can provide relevant information on the design of 
Table 4 . The summary of results obtained by the resolution of The MILP Mode

\begin{tabular}{|l|l|l|l|l|}
\hline Instance & $\begin{array}{l}\text { Problem Size } \\
n \times m \times k\end{array}$ & Makespan & GAP & $\begin{array}{l}\text { Resolution } \\
\text { Time }(s)\end{array}$ \\
\hline Inst 3.1 & $3 \times 3 \times 1$ & 221 & - & 0.05 \\
\hline Inst 3.2 & $3 \times 3 \times 2$ & 221 & - & 0.06 \\
\hline Inst 3.3 & $3 \times 3 \times 3$ & 221 & - & 0.06 \\
\hline Inst 4.1 & $4 \times 4 \times 1$ & 337 & - & 0.33 \\
\hline Inst 4.2 & $4 \times 4 \times 2$ & 337 & - & 0.64 \\
\hline Inst 4.3 & $4 \times 4 \times 3$ & 337 & - & 0.5 \\
\hline Inst 5.1 & $5 \times 5 \times 1$ & 433 & - & 0.5 \\
\hline Inst 5.2 & $5 \times 5 \times 2$ & 433 & - & 6.15 \\
\hline Inst 5.3 & $5 \times 5 \times 3$ & 433 & - & 5.6 \\
\hline Inst 6.1 & $6 \times 6 \times 1$ & 628 & - & 5.02 \\
\hline Inst 6.2 & $6 \times 6 \times 2$ & 623 & - & 82.2 \\
\hline Inst 6.3 & $6 \times 6 \times 3$ & 623 & - & 25.74 \\
\hline Inst 7.1 & $7 \times 7 \times 1$ & 709 & - & 30.04 \\
\hline Inst 7.2 & $7 \times 7 \times 2$ & 695 & - & 1106.94 \\
\hline Inst 7.3 & $7 \times 7 \times 3$ & $(773 ; 556)$ & $28.07 \%$ & 9236.43 \\
\hline Inst 8.1 & $8 \times 8 \times 1$ & $(812 ; 797)$ & $1.85 \%$ & 8113.05 \\
\hline Inst 8.2 & $8 \times 8 \times 2$ & $820 ; 713$ & $13,05 \%$ & 277100 \\
\hline Inst 8.3 & $8 \times 8 \times 3$ & $(833 ; 616)$ & $20.05 \%$ & 97100 \\
\hline Inst 9.1 & $9 \times 9 \times 1$ & $(1120 ; 931)$ & $20.35 \%$ & 7794 \\
\hline Inst 9.2 & $9 \times 9 \times 2$ & $(1080 ; 847)$ & $27,51 \%$ & 267531 \\
\hline Inst 9.3 & $9 \times 9 \times 3$ & $(1074 ; 778)$ & $38,04 \%$ & 255337 \\
\hline Inst 10.1 & $10 \times 10 \times 1$ & $(1295 ; 887)$ & $46,11 \%$ & 69420 \\
\hline Inst 10.2 & $10 \times 10 \times 2$ & $(1176 ; 791)$ & $48,67 \%$ & 93716 \\
\hline Inst 10.3 & $10 \times 10 \times 3$ & $(1211 ; 810)$ & $49,50 \%$ & 144164 \\
\hline & & & &
\end{tabular}

a robotic cell by measuring the benefits of adding additional resources.

\section{CONCLUSION}

In this work, the Job Shop Scheduling problem with Transportation and subject to No Wait and Blocking Constraints is described. An existing model from the literature is extended to show how an integer programming formulation can be applied to solve the problem. An algorithm for the construction of a heuristic based on avoidance of blocking situations rules is proposed. Experimental results for the proposed mathematical model are provided. These results show the limit of our proposed mathematical model to solve problems with large sizes. Future researches will be focused on a hybrid methods based on this article proposed construction heuristic. The aim of this methods is to produce, in a reasonable time frame, for problem with large sizes a solution that is not the best but is good enough for solving the problem. Experimental results will be established to show the efficiency of our developed methods comparing to exact methods.

\section{REFERENCES}

[1] A.S. Jain, S. Meeran, Deterministic job-shop scheduling: Past present and future, European Journal of Operational Research 113 (1999) 390-434.

[2] N.G. Hall, C. Sriskandarajah, A survey of machine scheduling problems with blocking and no-wait in process, Operations Research 44 (3) (1996) 510-525.

[3] O. Candar, Machine scheduling problems with blocking and no-wait in process, Working Paper [April-99], Department of Industrial Engineering, Bilkent University, Ankara, Turkey, 1999.
[4] Mascis, D. Pacciarelli, Machine scheduling via alternative graphs, Research Report, RT-DIA-46-2000, Italy, 2000.

[5] A. Mascis, D. Pacciarelli, Job-shop scheduling with blocking and no-wait constraints, European Journal of Operational Research 143 (2002) 498-517.

[6] Y. Mati, N. Rezg, X. Xie, Geometric approach and taboo search for scheduling flexible manufacturing systems, IEEE Transactions on Robotics and Automation 17 (6) (2001) 805-818.

[7] C.A. Brizuela, Y. Zhao, N. Sannomiya, No-wait and blocking job-shops: Challenging problems for GA's, IEEE 0-7803-77-2/ 01 (2001) 2349-2354.

[8] A. Klinkert, Optimization in design and control of automated high-density warehouses, Doctoral Thesis No. 1353, University of Fribourg, Switzerland, 2001.

[9] H. Groflin, A. Klinkert, Local search in job shop scheduling with synchronization and blocking constraints, Internal working paper [04-06], Department of Informatics, University of Fribourg, Switzerland, 2004.

[10] P. Brucker, T. Kampmeyer, Cyclic job shop scheduling problems with blocking,Ann. Oper. Res. 159 (2008) 161181.

[11] A. AitZai, B. Benmedjdoub, M. Boudhar, A branch and bound and parallel genetic algorithm for the job shop scheduling problem with blocking, Int. J. Oper. Res.14 (3) (2012) 343365.

[12] J. Grabowski, J. Pempera, Sequencing of jobs in some production system, European Journal of Operation Research 125 (2000) 535550.

[13] D. Pacciarelli, M. Pranzo, Production scheduling in a steelmaking-continuous casting plant, Computer and Chemical Engineering 28 (2004) 28232835.

[14] J. Romero, L. Puigjaner, T. Holczinger, F. Friedler, Scheduling intermediate storage multipurpose batch plants using the S-graph, AIChE J. 50 (2004) 403417.

[15] L. Chen, N. Bostel, P. Dejax, J. Cai, L. Xi, A tabu search algorithm for the integrated scheduling problem of container handling systems in a maritime terminal, European Journal of Operation Research 18 (2007) 4058.

[16] A. DAriano, D. Pacciarelli, M. Pranzo, A branch and bound algorithm for scheduling trains in a railway network, European Journal of Operation Research 183 (2007) 643657.

[17] J. Hurink ,S. Knust, Tabu search algorithms for job-shop problems with a single transport robot, European Journal of Operational Research 2005;162(1):99111.

[18] J. Blazewicz,H. Eiselt ,G. Finke,G. Laporte,J. Weglarz, Scheduling tasks and vehicles in a flexible manufacturing system, International Journal of Flexible Manufacturing Systems 1991;4(1):516.

[19] U. Bilge,G. Ulusoy, A time window approach to simultaneous scheduling of machines and material handling system in an FMS. Operations Research 1995;43(6):105870.

[20] G. Ulusoy, F. Sivrikaya-erifolu, U. Bilge, A genetic algorithm approach to the simultaneous scheduling of machines and automated guided vehicles, Computers \& Operations Research 1997;24(4):33551.

[21] G. Alvarenga,G. Mateus, G. De Tomi, A genetic and set partitioning two-phase approach for the vehicle routing problem with time windows, Computers \& Operations Research 2007;34(6):156184. 
[22] M. Soylu,N. O zdemirel, S. Kayaligil, A self-organizing neural network approach for the single AGV routing problem, European Journal of Operational Research 2000;121(1):12437.

[23] J. Hurink,S. Knust, A tabu search algorithm for scheduling a single robot in a job shop environment, Discrete Applied Mathematics 2002;119(12):181203.

[24] T. Abdelmaguid,A. Nassef, B. Kamal, M. Hassan, A hybrid GA/heuristic approach to the simultaneous scheduling of machines and automated guided vehicles, International Journal of Production Research 2004;42(2):26781.

[25] P. Lacomme,M. Larabi, N. Tchernev, A disjunctive graph for the job shop with several robots, In: MISTA conference; 2007. p. 28592.

[26] L. Deroussi, M. Gourgand, N. Tchernev, A simple metaheuristic approach to the simultaneous scheduling of machines and automated guided vehicles, International Journal of Production Research 2008;46(8):214364.

[27] H. Gong, L. Tang, Two-machine flow shop scheduling with intermediate transportation under job physical space consideration, Computers \& Operations Research 2011;8(9):126774.

[28] M. Mateo, R. Companys, J. Bautista, Resolution of graphs with bounded cycle time for the cyclic hoist scheduling problem, In: 8th International Workshop on Project Management and Scheduling. Cite seer; 2002. p. 25760.

[29] A. Caumond, P. Lacomme, A. Moukrim, N. Tchernev, An MILP for scheduling problems in an FMS with one vehicle, European Journal of Operational Research 2009;199(3):70622.

[30] Y. Crama, J. Van de Klundert, Cyclic scheduling of identical parts in a robotic cell. Operations Research 1997;45(6):95265.

[31] N. Brauner, G. Finke, V. Lehoux-Lebacque, C. Potts, J. Whitehead, Scheduling of coupled tasks and one machine no wait robotic cells, Computers \& Operations Research 2009;36(2):3017.

[32] AI. Corra, A. Langevin, L-M. Rousseau, Scheduling and routing of automated guided vehicles: a hybrid approach. Computers \& Operations Research 2007; 34(6):1688707, [part special Issue: Odysseus 2003 second international workshop on freight transportation logistics. doi:10.1016/j.cor.2005.07.004].

[33] E. Stafford, F. Tseng,J. Gupta, Comparative evaluation of MILP flow shop models, Journal of Operations Research Society $2005 ; 56 ; 88101$.

[34] A. Manne, On the job shop scheduling problem. Operations Research 1960;8,21922 